\title{
Mortalidade por câncer de próstata no estado do Piauí: perfil epidemiológico e tendências
}

\author{
Mortality due to prostate cancer in the state of Piauí: epidemiological profile and trends \\ Mortalidad por cáncer de próstata en el estado de Piauí: perfil epidemiológico y \\ tendencias
}

Lorrana Monteiro Santos ${ }^{1}$, Jamily Cavalcante Gomes ${ }^{1}$, Kécya Patricia Costa Macêdo ${ }^{1}$, Felipe da Silva Carvalho', Lorrayne Monteiro Santos ${ }^{1}$, Adauto Teodoro Aguiar Neto', Lízia Carreiro Tomaz Aguiar', Aline Suelen Silva Nunes², Jéssica Larissa Sousa $\mathrm{Vaz}^{2}$, Roseane Mara Cardoso Lima Verde $^{3}$, Francisco das Chagas Araújo Sousa ${ }^{4}$, Leonardo Ferreira Soares ${ }^{5}$, Francisco Adalberto do Nascimento Paz ${ }^{1}$, Evaldo Hipólito de Oliveira ${ }^{2 *}$.

\section{RESUMO}

Objetivo: Analisar a frequência do câncer de próstata no Piauí, no período de 2007 a 2016. Métodos: Tratase de estudo retrospectivo, onde a coleta dos dados ocorreu na base de dados DATASUS (Departamento de Informática do SUS), no Sistema Informação de Mortalidade (SIM), baseado nos dados estatísticos populacional do IBGE 2010 e processados no Microsoft Excel 2010. Os dados estatísticos foram realizados a partir de cálculos de análises de tendência baseados nas variáveis dependentes (y) que foram as taxas por ano de mortalidade e independente (x) considerados os anos de estudo. Resultados: No Piauí, entre os anos de 2007 a 2016 obteve-se cerca de 2281 casos de morte por Câncer de próstata, onde a os idosos com mais de 80 anos, pardos possuem maior prevalência. Conclusão: Tendo esse estudo fortalecido à importância da realização dos testes de prevenção e diagnóstico precoce, além de servido como instrumento de análise dos índices de letalidade por câncer de próstata.

Palavras-chave: Epidemiologia, Mortalidade por câncer, Câncer de próstata.

\begin{abstract}
Objective: To analyze the frequency of prostate cancer in Piauí, from 2007 to 2016. Methods: This is a retrospective study, where the data collection was performed in the DATASUS database (Department of Information Technology of SUS), in the Information System of Mortality (SIM), based on the population statistics of IBGE 2010 and processed in Microsoft Excel 2010. Statistical data were calculated based on trend analysis based on the dependent variables $(y)$, which were rates per year of mortality and independent $(x)$ considered the year of study. Results: In Piaui, between the years of 2007 and 2016, there were approximately 2281 cases of death due to prostate cancer, where the elderly with more than 80 years of age, pardos have a higher prevalence. Conclusion: This study strengthened the importance of performing the tests of prevention and early diagnosis, besides being used as an instrument for the analysis of the lethality indexes for prostate cancer.
\end{abstract}

Key words: Epidemiology, Cancer mortality, Prostate cancer.

\section{RESUMEN}

Objetivo: Analizar la frecuencia del cáncer de próstata en Piauí, en el período de 2007 a 2016. Métodos: Se trata de un estudio retrospectivo, donde la recolección de los datos ocurrió en la base de datos DATASUS (Departamento de Informática del SUS), en el Sistema Información de Mortalidad (SIM), basado en los datos

${ }^{1}$ Centro Universitário Santo Agostinho. Teresina - PI.

²Universidade Federal do Piaú. Teresina - PI *E-mail: evaldohipolito@gmail.com

${ }^{3}$ Doutoranda em Engenharia Biomédica - PPGEB, Universidade Brasil, São Paulo - SP.

${ }^{4}$ Universidade Estadual do Piauí. Teresina - PI.

5Universidade Estadual da Paraíba. João Pessoa - PB. 
estadísticos poblacionales del IBGE 2010 y procesados en Microsoft Excel 2010. Los datos estadísticos se realizaron a partir de cálculos de análisis de tendencia basados en las variables dependientes $(\mathrm{y})$ que fueron las tasas por año de mortalidad e independiente $(x)$ considerados los años de estudio. Resultados: En Piaui, entre los años 2007 a 2016 se obtuvieron cerca de 2281 casos de muerte por Cáncer de próstata, donde a los ancianos con más de 80 años, pardos poseen mayor prevalencia. Conclusión: Teniendo ese estudio fortalecido a la importancia de la realización de las pruebas de prevención y diagnóstico precoz, además de servir como instrumento de análisis de los índices de letalidad por cáncer de próstata

Palabras clave: Epidemiología, Mortalidad por cáncer, Cáncer de próstata.

\section{INTRODUÇÃO}

A morbimortalidade do câncer de próstata $(C P)$ ou cranco de próstata nas últimas décadas vem sendo reconhecido como um grave transtorno a saúde pública (GOMES CHR, et al., 2008). No Brasil, o CP é o segundo câncer mais presente nos homens, estando após o câncer de pele não-melanoma (QUIJADA PSD, et al., 2017).

A próstata é uma glândula constituída de células, no qual inclui-se da seção do sistema genital masculino, tendo como função gerar e armazenar um fluido que em agrupamento com o fluido seminal e os espermatozoides formam o sêmen (JÚNIOR, BACELAR; MENEZES, 2015). A multiplicação desordenada dessas células da glândula provoca o câncer fazendo com que ela endureça, além de em etapa inicial não desenvolvendo sintomas aparentes, por isso o exame preventivo é fundamental (GOMES CHR, et al., 2008).

A biopsia é o exame confirmatório para $\mathrm{CP}$, feito após exames como o toque retal (TR) e do doseamento do antígeno prostático específico (PSA). O TR verifica um dos primeiros sintomas aparentes do CP que é o endurecimento da mesma, onde após esse exame o médico verifica a precisão de novos para confirmação do diagnostico (DE SOUSA, et al., 2011). Ainda que a fase inicial do CP não é observado em muitos quadros sintomas, em alguns casos podem aparecer e devem ser levados em consideração como disúria, dificuldade de urinar ou interromper a urina, vontade de urinar imediatamente, e fazer força ao urinar, sensação de dor abaixo dos testículos, sangue na urina ou esperma (RHODEN EL, AVERBECK MA, 2009).

Alguns dos sintomas presentes em estágios avançados de CP são a disúria, dificuldade de urinar ou interromper a urina, vontade de urinar imediatamente, e fazer força ao urinar, sensação de dor abaixo dos testículos, sangue na urina ou esperma (RHODEN EL, AVERBECK MA, 2009), diante desses sintomas devese procurar auxilio medico para testes que auxiliam num diagnóstico.

Idade, hereditariedade e origem étnica são alguns dos elementos relacionados ao CP sendo necessárias medidas preventivas tem como finalidade diminuir a incidência das doenças nas populações. Após diagnostico o tratamento é iniciado de forma individual, sendo levada em consideração a idade do paciente, capacidade da próstata, etapa histológico e estágio de câncer, podendo ser radioterapia, terapias endócrinas e até cirurgias (GOMES CHR, et al., 2008).

Esse estudo tem como objetivo analisar a frequência do câncer de próstata no Piauí, no período de 2007 a 2016. Além de descrever os métodos de prevenção e diagnostico do câncer de próstata.

\section{MÉTODOS}

Estudo retrospectivo realizado entre os anos de 2007 a 2016 no estado do Piaui. A busca dos dados da foi realizada nas bases de dados da Medline (Medical Literature Analysis and Retrieval System Online) e Scielo (Scientific Electronic Library Online) apenas em artigos em língua portuguesa publicados entre 20002019 usando como descritores: cranco de próstata, câncer de próstata, glândula da próstata. As faixas etárias estudados foram pacientes de 15 a 49, 50 a 59,60 a 69,70 a 79 e pessoas com 80 ou mais.

A coleta dos dados ocorreu na base de dados DATASUS (Departamento de Informática do SUS), no Sistema Informação de Mortalidade (SIM), baseado nos dados estatísticos populacional do IBGE 2010 e processados no Microsoft Excel 2010. Os dados estatísticos foram realizados a partir de cálculos de análises 
de tendência baseados nas variáveis dependentes ( $\mathrm{y}$ ) que foram, as taxas por ano de mortalidade e independente $(\mathrm{x})$ considerando os anos de estudo. Devido os dados dessa pesquisa ser de caráter público, não demandou autorização do comitê de ética e pesquisa em seres humanos.

\section{RESULTADOS E DISCUSSÃO}

O câncer de próstata é um grande obstáculo de saúde mundial, devido a grande frequência e o aumento com que vem ocorrendo nos últimos anos, sendo considerada a sexta forma de câncer mais popular no mundo (PINTO DE MEDEIROS A, et al., 2011). No Piauí, entre os anos de 2007 a 2016 obtiveram-se cerca de 2.281 de casos de morte por CP, onde o ano de 2015 atingiu maior prevalência. Segundo os dados analisados houve uma tendência crescente de letalidade por CP, como mostra o Gráfico 1, esses dados podem estar relacionados ao grande atrito dos homens ao exame preventivo de TR (EL BAROUKI MP, et al., 2012).

$\mathrm{O}$ TR serve como método de rastreio para o $\mathrm{CP}$, onde a analise evidenciou maior prevalência em idosos com mais de 80 anos, seguida de idosos com 70 a 79 anos (Gráfico 2), por isso preconiza-se que adulto com mais de 50 anos de idade ou com histórico familiar de câncer, procure anualmente um urologista para realizar o teste de prevenção, pois de início o câncer aparece assintomático e normalmente quando se tem queixa já está em estágio avançado (MODESTO AAD, et al., 2017).

Gráfico 1 - Taxa bruta de mortalidade segundo o ano no Piauí, entre 2007 - 2016.

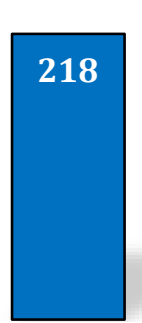

2007

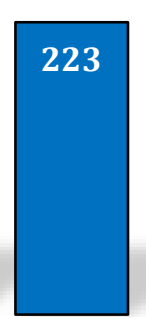

2008

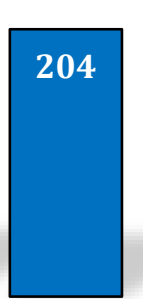

2009

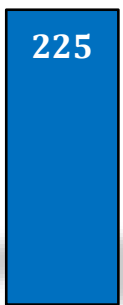

2010

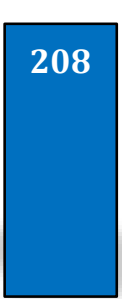

2011

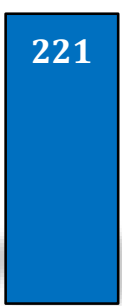

2012

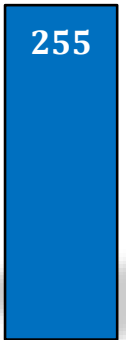

2013

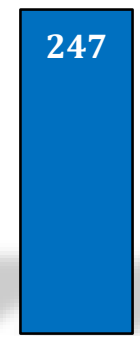

2014

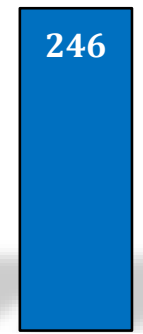

2015

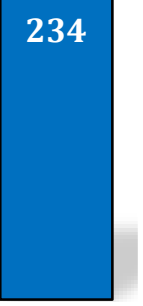

2016

Fonte: Santos LM, et al., 2019. Baseado em: MS/SVS/CGIAE - Sistema de Informações sobre Mortalidade - SIM.

Gráfico 2 - Mortalidade no Piauí de câncer de próstata, segundo idade. Período: 2007-2016

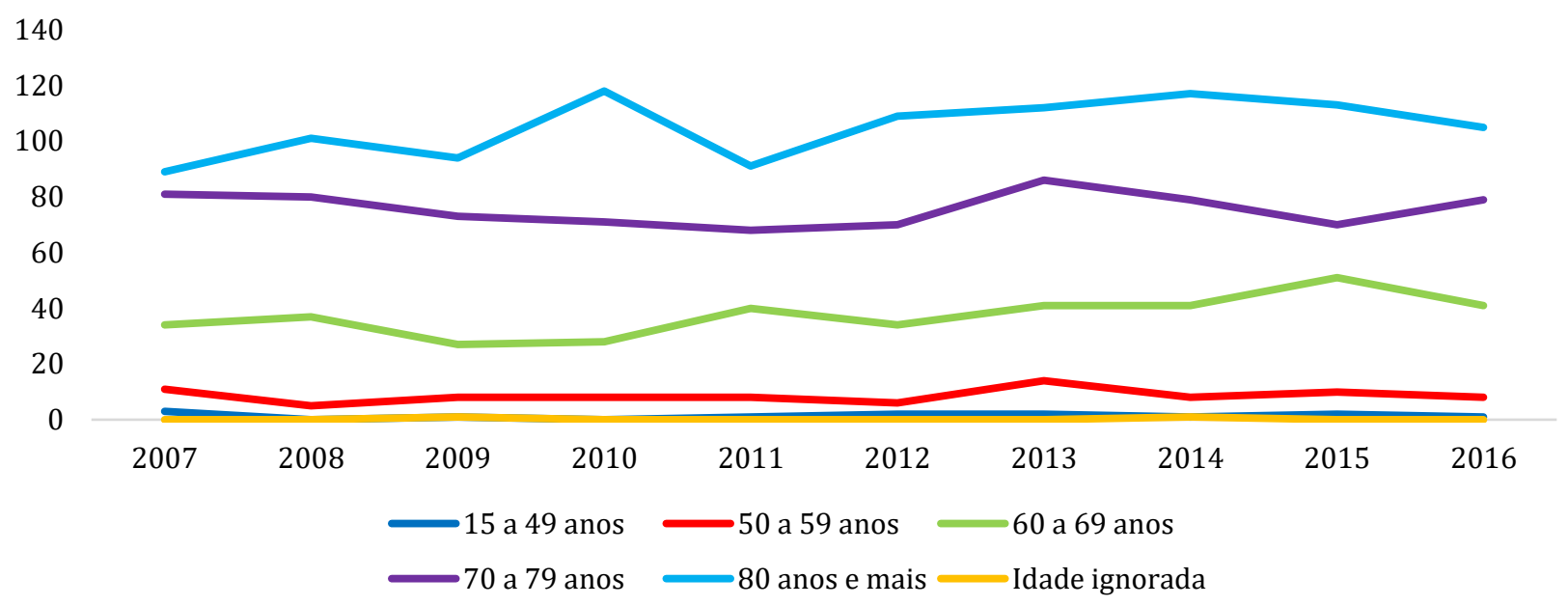

Fonte: Santos LM, et al., 2019. Baseado em: MS/SVS/CGIAE - Sistema de Informações sobre Mortalidade SIM. 
A maior prevalência entre idosos pode estar relacionado ao fato da pouca busca pelos exames preventivos que normalmente diagnosticam o câncer em estágio inicial, e quando o paciente busca tratamento relatando sintomas o câncer já está em estágio avançado tornando mais complicado o tratamento e chances de cura (OLIVEIRA PSD, 2015).

Nessa mesma visão, as objeções para busca de precaução e diagnóstico precoce pode estar relacionado ao preconceito masculino, pela busca da realização do teste retal ou ainda ou simultaneamente com o pouco acesso a rede assistencial de saúde, bem como outros fatores, que aumentam a mortalidade no Piauí de CP, visto que muitos casos só são diagnosticados quando não se tem mais chances de tratamento (KNOB PINTO B, 2014; OLIVEIRA PSD, 2015). Com o crescimento populacional e maior possibilidade de vida, cresce o número de doenças entre os idosos, representando não só um grande problema a saúde publica mais como grande agravamento socioeconômico (BRITO ALF, 2016).

$A$ idade, hereditariedade e origem étnica, são alguns dos elementos relacionados ao $\mathrm{CP}$, onde o estudo mostrou maior letalidade em pardos (Gráfico 3) (EL BAROUKI MP, et al., 2012). Já é sabido a maior predisposição de negros a doenças como $\mathrm{CP}$, onde o estudo demostrou que a maior frequência esta na cor parda, podendo esta relacionado a grande miscigenação brasileira, tornando outras cores/raças mais pré disponível a doenças, visto que existe uma predisposição genética nesses indivíduos.

Gráfico 3 - Proporção de óbitos por câncer de próstata no estado do Piauí, segundo o ano de notificação e raça/cor.

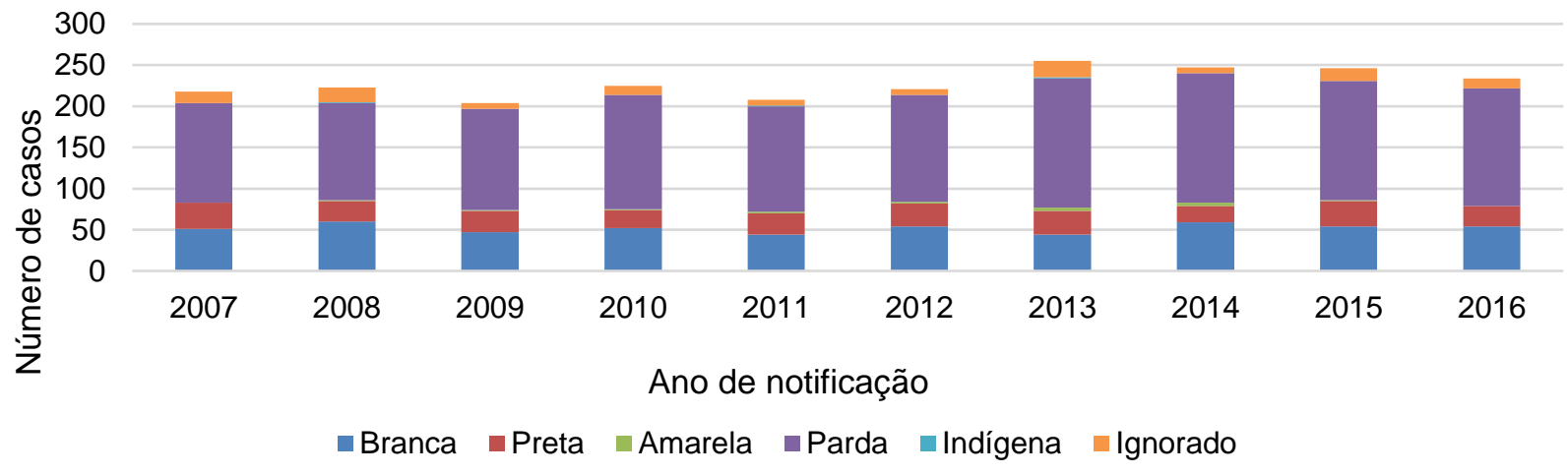

Fonte: Santos LM, et al., 2019. Baseado em: MS/SVS/CGIAE - Sistema de Informações sobre Mortalidade SIM.

Gráfico 4 - Taxa de mortalidade ( $\left.{ }^{*} 100.000\right)$ por câncer de próstata e linha de tendência no estado do Piauí, no período de 2007 a 2016.

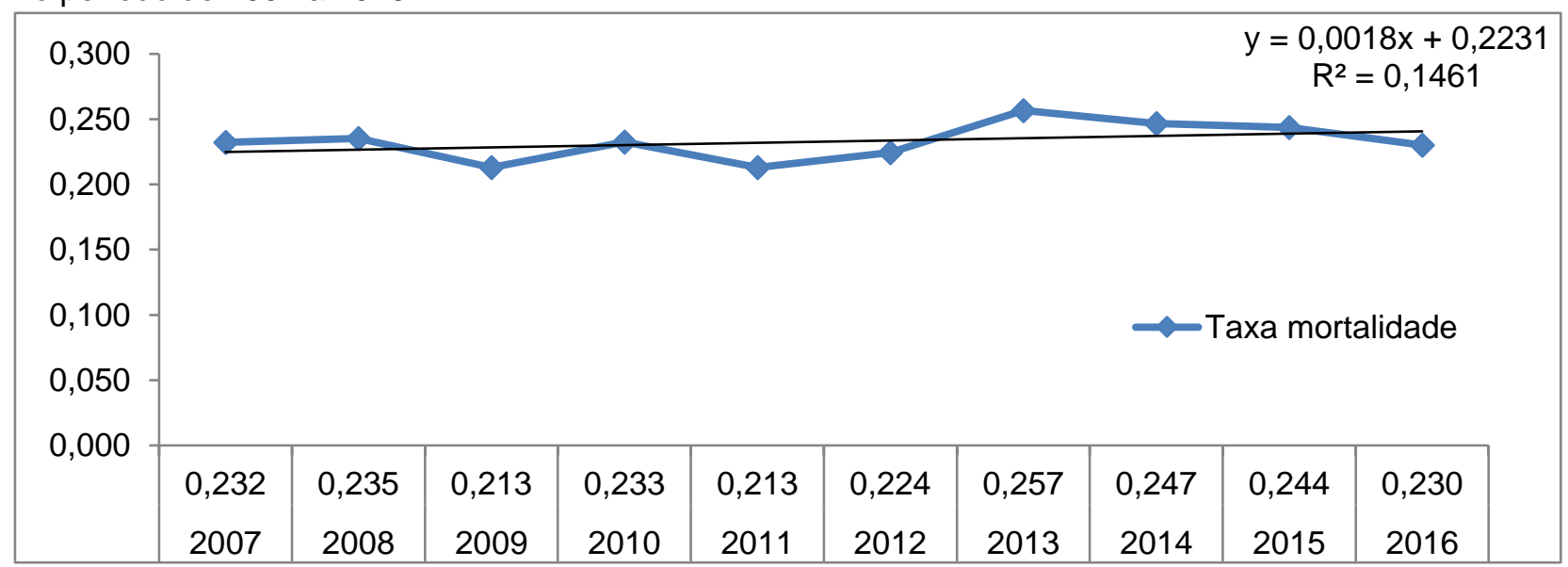

Fonte: Santos LM, et al., 2019. Baseado em: MS/SVS/CGIAE - Sistema de Informações sobre Mortalidade - SIM. 
Os dados do Gráfico 4 mostram sobre o ponto de vista da linha de tendência mostra instabilidade de número de casos de $\mathrm{CP}$, com elevações e declínios nos anos estudados, baseado nas estatísticas $\mathrm{y}=$ $0,0018 x+0,2231, R^{2}=0,1461$, comparado ao com o estudo realizado por Ferreira DB e Mattos IE (2015) sobre o câncer de mama acometido em mulheres, evidencia a maior frequência de CP do que de câncer de mama visto que os dados demonstraram estabilidade no número de casos de letalidade por câncer de mama, dependentes dos estados brasileiros.

\section{CONCLUSÃO}

Portanto, pode-se observar com esse estudo um aumento significativos dos índices de mortalidade por câncer de próstata no Piauí, com cerca de 2281 casos nos anos analisados (2007 a 2016), demonstrando a necessidade de intervenções e prevenção do $\mathrm{CP}$, além de devida orientação aos pacientes, pessoas com mais idade, buscando desmitificar mitos impostos pela sociedade, quebrando preconceitos contra os testes preventivos e de confirmatórios, visto que alguns dessas ocorrências podem intervir no diagnostico e tratamento, sendo considerada uma barreira sociocultural, influenciando num menor cuidado a esses pacientes, e maior exposição ao CP, fortalecendo a ideia de que a equipe multiprofissional é essencial no processo de auxilio desses pacientes, contribuindo a melhor assistência de saúde.

\section{REFERÊNCIAS}

1. BRITO ALF. Aumento da adesão ao rastremento do câncer de próstata: projeto de intervenção na área de abrângência da equipe de Saúde da Família Providência, Pará de Minas-MG. 2016.

2. DE SOUZA LM. Um toque na masculinidade: a prevenção do câncer de próstata em gaúchos tradicionalistas. Revista Gaúcha de Enfermagem, 2011; 32(1): 151, 2011.

3. EL BAROUKI MP. Rastreamento do câncer de próstata em homens acima de 50 anos através do exame diagnóstico de PSA. Revista Eletrônica Gestão \& Saúde, 2012; 3(2): 425-437.

4. FERREIRA DB, MATTOS IE. Tendência da mortalidade por câncer de mama em mulheres no estado do Rio de Janeiro, Brasil, 1996-2011. Cien Saude Colet, 2015; 20(3): 895-903.

5. GOMES CHR, et al. Avaliação do conhecimento sobre detecção precoce do câncer dos estudantes de Medicina de uma universidade pública. Rev bras cancerol, 2008; 54(1): 25-30.

6. GOMES R, et al. A prevenção do câncer de próstata: uma revisão da literatura. Ciência \& Saúde Coletiva, 2008; 13: 235-246.

7. GOMES R, et al. As arranhaduras da masculinidade: uma discussão sobre o toque retal como medida de prevenção do câncer prostático. Ciência \& saúde coletiva, 2008; 13: 1975-1984.

8. JÚNIOR B, MENEZES CS. Câncer de próstata: métodos de diagnóstico, prevenção e tratamento. Brazilian Journal of Surgery and Clinical Research. Minas Gerais, 2015; 10(3): 40-46.

9. KNOB PINTO B, et al. Identidade do homem resiliente no contexto de adoecer por câncer de próstata: uma perspectiva cultural. Revista Brasileira de Enfermagem, 2014; 67(6).

10. MODESTO AAD, et al. Um novembro não tão azul: debatendo rastreamento de câncer de próstata e saúde do homem. Interface-Comunicação, Saúde, Educação, 2017.

11. OLIVEIRA PSD, et al. Percepção dos homens sobre o exame toque retal. Rev. enferm. UFPE on line, 2015; 9(5): 7760-7765.

12. PINTO DE MEDEIROS A, et al. Fatores de risco e medidas de prevenção do câncer de próstata: subsídios para a enfermagem. Revista Brasileira de Enfermagem, 2011; 64(2).

13. QUIJADA PDS, et al. Câncer de próstata: retrato de uma realidade de pacientes em tratamento. Rev. enferm. UFPE on line, 2017; 11 (n. supl. 6): 2490-2499.

14. RHODEN EL, AVERBECK MA. Câncer de próstata e testosterona: riscos e controvérsias. Arq Bras Endocrinol Metab, 2009; 53(8): 956-962. 